\title{
EXAMINATION OF THE SKIN CONDUCTANCE LEVEL (SCL) AS AN INDEX OF THE ACTIVITY OF THE SYMPATHETIC NERVOUS SYSTEM FOR APPLICATION IN THE CONTINUOUS BLOOD PRESSURE MEASUREMENT
}

\author{
Janina Horstick, Simon Siebers, Claus Backhaus \\ Center of ergonomics and medical engineering, \\ University of Applied Sciences Münster, Münster, Germany
}

\begin{abstract}
This paper displays standardized measurements to examine the skin conductance level (SCL) as an index for the sympathetic nervous system's activity considering a possible standardization of personal variability. SCL measurements were performed at baseline, Cold-Pressor-Tests and Stroop Tests for 15 subjects and inter- and intra-individual mean SCL values were examined. The logarithmic representation displays a high inter-individual variability, denoted by a high fluctuation range of the absolute values and SCL amplitudes. A standardization of inter-individuality could not be achieved by the applied methods (range correction and z-transformation). For the relative intra-individual comparison of the stress situation to the baseline, a method of correction for the determination of the real effect of sympathetic activation was established. EDA should be combined with other parameters of the ANS for a more precise evaluation of stress situations in the context of changes in blood pressure.
\end{abstract}

\section{KEYWORDS}

Electrodermal activity, Sympathetic nervous system, Continuous blood pressure, Patient monitoring

\section{SITUATION}

Continuous high blood pressure is the No. 1 risk factor for cardiovascular diseases [1], because the asymptomatic course of hypertension can remain unrecognized until initial organ damage arises. The chronic activity of the sympathetic nervous system, a subsystem of the ANS, can be considered a critical risk factor of hypertension. Due to the ergotropic effect of the sympathetic nervous system, each sympathetic activation can result in an increase of blood pressure.

For the retrospective analysis of blood pressure data, it is useful to measure sympathetic nervous activity as well as continuous blood pressure. Blood pressure fluctuations can then be compared with possible sympathetic activities. While an increased blood pressure under physical exertion is quite common, it is more likely to be pathological under normal conditions, meaning that is when there are no cognitive, mental or emotional triggers. To allow for such analysis a device was designed that can measure both blood pressure and electrodermal activity (EDA) simultaneously. This device is a portable blood pressure monitor that enables an in-depth analysis with arterial blood pressure measurement on the finger according to Peñáz [2] method. 
International Journal of Biomedical Engineering and Science (IJBES), Vol. 5, No. 2, April 2018

Electrodermal activity has become a psychophysiological standard method for the measurement of sympathetic activity [3]. Because of the integration of central nervous processes into the vegetative sweat gland activation, EDA reflects primarily psychophysical activations [4]. Thus, EDA is an appropriate indicator of psychological states. It can be influenced by both external and internal factors; it reflects changes in mood, by immediately and sensitively changing. Some authors describe an influence of temperature, gender or age on EDA measurement [3, 5-7] whose reliability is not established in all studies. It is therefore difficult to compare the studies in order to define a consensus. It is certain that EDA has high intra- and inter-individual fluctuations due to permanent variations in people's moods and changes of environmental factors.

The SCL (skin conductance level) and SCR (skin conductance response) are two characteristic parameters of EDA that represent phasic and tonic components. The SCRs are short, phasic electrodermal responses that increase within one second after a discrete stimulus (Figure 1). The tonic component of skin conductivity is the skin conductivity level (SCL). A slight change in the value range is characteristic for this parameter. The absolute values of the SCL can vary from 2 to $100 \mu \mathrm{S}$, yet have a mainly individual continuity. Reasons for increases in the skin conductance level are multivariate: Both cognitive and emotional stress triggers a response from the SCL. Therefore, this "basic measure" is also referred as the general "arousal" of a person $[3,4,8]$.

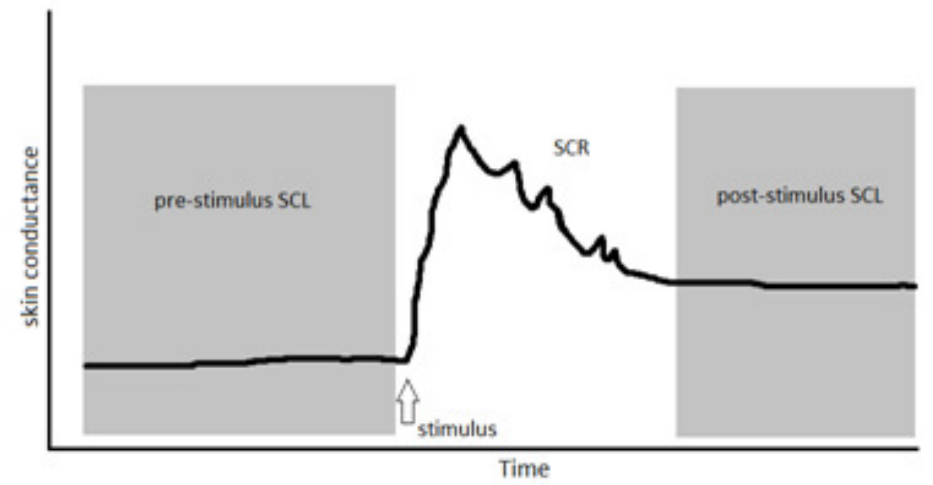

Figure 1. Smooth SCL (tonic phase) is interrupted by a rapid rise in skin conductance (SCR, or phasic phase) due to a stimulus. Subsequently, the skin conductance attains a new SCL, which is usually higher than the pre-stimulus level. Adapted from [9]

Furthermore, skin conductive responses can be spontaneous and stimulus independent, called non-specific skin-conduction reactions (NS.SCR). The frequency of the NS.SCRs is determined under resting conditions and is a tonic parameter that indicates the stress state of a person.

The continuous monitoring of blood pressure and EDA combined with data analysis to extract the desired clinical information can avoid an unnecessary and potentially harmful therapy or the omission of a required therapy. However, monitoring EDA as a significant biofeedback for sympathetic activity requires a comparable evaluation of the EDA data. The major problem when it comes to the comparison is the high personal variability of the EDA data. On the one hand, it is caused by physiological properties, on the other hand by a variety of technical methods. Against this background EDA is measured under own standardized conditions in order to examine the data for intra- and inter-individual comparability / standardization possibilities.

\section{METHODS}

Only healthy, male and female volunteers aged between 18 and 45 years were included in the test series. The recruiting of the subjects took place via direct approach of persons studying or working at the Fachhochschule Münster. 
International Journal of Biomedical Engineering and Science (IJBES), Vol. 5, No. 2, April 2018

\subsection{Procedure}

A standardized series with three test conditions, a relaxing situation (baseline) and two different stress situations, was carried out to investigate the personal variability.

For a relative analysis of changes in EDA, the stress situations are compared with the baseline. The baseline was recorded for 10 minutes for each person at the beginning of the test series: Participants were asked to sit quietly on a chair. Subsequently, physical (cold-pressor-test [12, 13]) and cognitive (Stroop-test [14]) stimuli were applied for three minutes each and repeated three times. Before the first stress stimulus was introduced, a rest measurement of five minutes was performed and between the repetitions, two minutes of rest were measured.

For the physical stress situation (cold-pressor-test), the subjects were asked to hold their hand (up to the wrist) in cold ice water $\left(0-4{ }^{\circ} \mathrm{C}\right)$. In the cognitive stress test (Stroop-test), participants were shown color words presented in colors that were or were not congruent to the color word. In the Stroop-test variant used [15], each word was presented for 3 seconds with an inter-stimulus interval of 2 second. Each word indicated one of four colors (red, green, yellow or blue) and was simultaneously identified in one of these colors.

The baseline measurement was followed by the Stroop-test for the half of the subjects and the cold-pressor-test for the other half. The assignment to the respective test series was randomized. The test was performed in a quiet room with a constant temperature of $22{ }^{\circ} \mathrm{C}$ at two different times (between 10:00 - 12:00 and 13:30 - 15:30).

\subsection{Measures}

The EDA measurements were performed with the Biopac MP150® system and a sampling rate of $2 \mathrm{kHz}$. Pre-gelled non-polarizable silver / silver chloride electrodes and a constant voltage of 0.5 $\mathrm{V}$ were used. Without pretreatment, the non-dominant hand and the hypothenic electrodes placements were selected.

\subsection{Data Processing}

The baseline measurements were compared with stress situations to analyze the interindividuality of the SCL values among a sample. Regarding the skin conductance level (SCL), Schell et al. [10] showed temporal stability over a 1-year period in schizophrenia patients $(r=.43)$ and healthy persons $(r=.61)$ and El-Sheik [11] showed long-term stability $(r=.30)$ of SCL in children. In addition repeated measurements were compared intra-individually to reveal the reliability of a stress stimulus for possible use as a calibration measure for EDA recording.

Table 1. Parameters used and description of collection method.

\begin{tabular}{|l|l|}
\hline Parameter & Collection \\
\hline SCL & Entire mean of all data in a time interval without artifacts \\
\hline $\begin{array}{l}\text { Amplitude of } \\
\text { SCLs }\end{array}$ & $\begin{array}{l}\text { Difference between mean SCL values of the strain stimulus and mean SCL values of } \\
\text { immediately preceding rest measurements }\end{array}$ \\
\hline NS.SCRs & $\begin{array}{l}\text { Number of NS.SCRs per minute. The NS.SCRs frequency is measured under resting } \\
\text { conditions in absence of extern stimuli }\end{array}$ \\
\hline
\end{tabular}


International Journal of Biomedical Engineering and Science (IJBES), Vol. 5, No. 2, April 2018

For the analysis the mean of the skin conductivity level (SCL) was determined over the experimental episodes (baseline, 3x Stroop-tests, 3x cold-pressor-test). For the baseline the last 5 minutes were chosen. The study was based on the recommended correction procedures of the "Guidelines for the Analysis of EDA and SCR in Psychological Experiments" with the use of Biopac and the AcqKnowledge ${ }^{\circledR}$ Software by Braithwaite et al. [16]. To preprocess and delete artefacts in the data, the sampling rate was reduced to $62.5 \mathrm{~Hz}$ and smoothed by a median filter. Subsequently, a low-pass filter of $1 \mathrm{~Hz}$ was used to determine the tonic EDA component. For the detection of spontaneous fluctuations (NS.SCRs) and SCRs, an amplitude criterion of $0.05 \mu \mathrm{S}$ was chosen. If no spontaneous fluctuations could be found throughout the entire measurement, subjects were excluded as "non-responder" and the remaining data set was evaluated selectively.

Two established correction methods were used to reduce the high variability. According to Lykken and colleagues $[17,18,19]$, the range correction is used to determine the individual range by representing the individually measured values in relation to the respective SCL range. The minimum and maximum SCL values of a person are determined. An alternative standardization method is provided by Ben-Shakhar [20] who suggest transforming original values into z-values with a defined mean value and standard deviation to make the sample values comparable (ztransformation).

\subsection{Statistical Analysis}

All computations were carried out using the SPSS statistical package (Version 24). The raw data, the arithmetic mean value and the transformed data $(\log )$ were used for analyses. The descriptive analysis of the collected measurements was done by exploratory data analysis. The mean (M) and the standard deviation (SD) were determined for each measure.

The data were examined for normal distribution (Shapiro-Wilk-Test) and sphericity (MauchlyTest). A significant Mauchly test was corrected by Greenhouse-Geisser.

Due to the fact that most of the physiological variables were skewed, average scores of the absolute data had to be transformed by the logarithmic transformation $(y=\log )$ to approximate the data to normal distribution. After this transformation, all variables with the exception of the skin temperature were normally distributed.

For the inferential statistical analysis of the data, one-way analyses of variance (ANOVA) with repeated measures were selected. If the ANOVA revealed significant differences in mean values, t-tests for dependent samples were used for further discrimination. The permissible probability of error is set at $5 \%$. For multiple comparisons (t-tests for dependent random samples and post-hoc tests) the significance level was adjusted according to the Bonferroni method. Therefore, the significance level was set at $\mathrm{p} \leq .02(\mathrm{p}=0.05$ divided by 3 [comparisons]).

For the study of possible correlations between the psychophysiological measures, Pearson's correlation coefficient was chosen for normally distributed data and the Spearman correlation coefficient for non-normally distributed data analyzed by the Shapiro-Wilk-Test [21].

\section{RESULTS}

A total of 15 people (10 males, 5 females) participated with a mean age of 27.1 (standard deviation 3.7). After checking the participation criteria, one subject was excluded from the further analyzes due to dyschromatopsia $(\mathrm{N}=14)$. 
International Journal of Biomedical Engineering and Science (IJBES), Vol. 5, No. 2, April 2018

Figure 2 shows the measurements for 14 subjects for the Stroop-test. A high inter-individual variability can be noticed, which is characterized by a high SCL fluctuation range of the absolute SCLs $(2-50 \mu \mathrm{S})$ and SCL amplitudes (0.1-17 $\mu \mathrm{S})$. The logarithmized values were in the range of $0-1.7$ for the SCL level and 0.02-1.7 for the amplitudes.

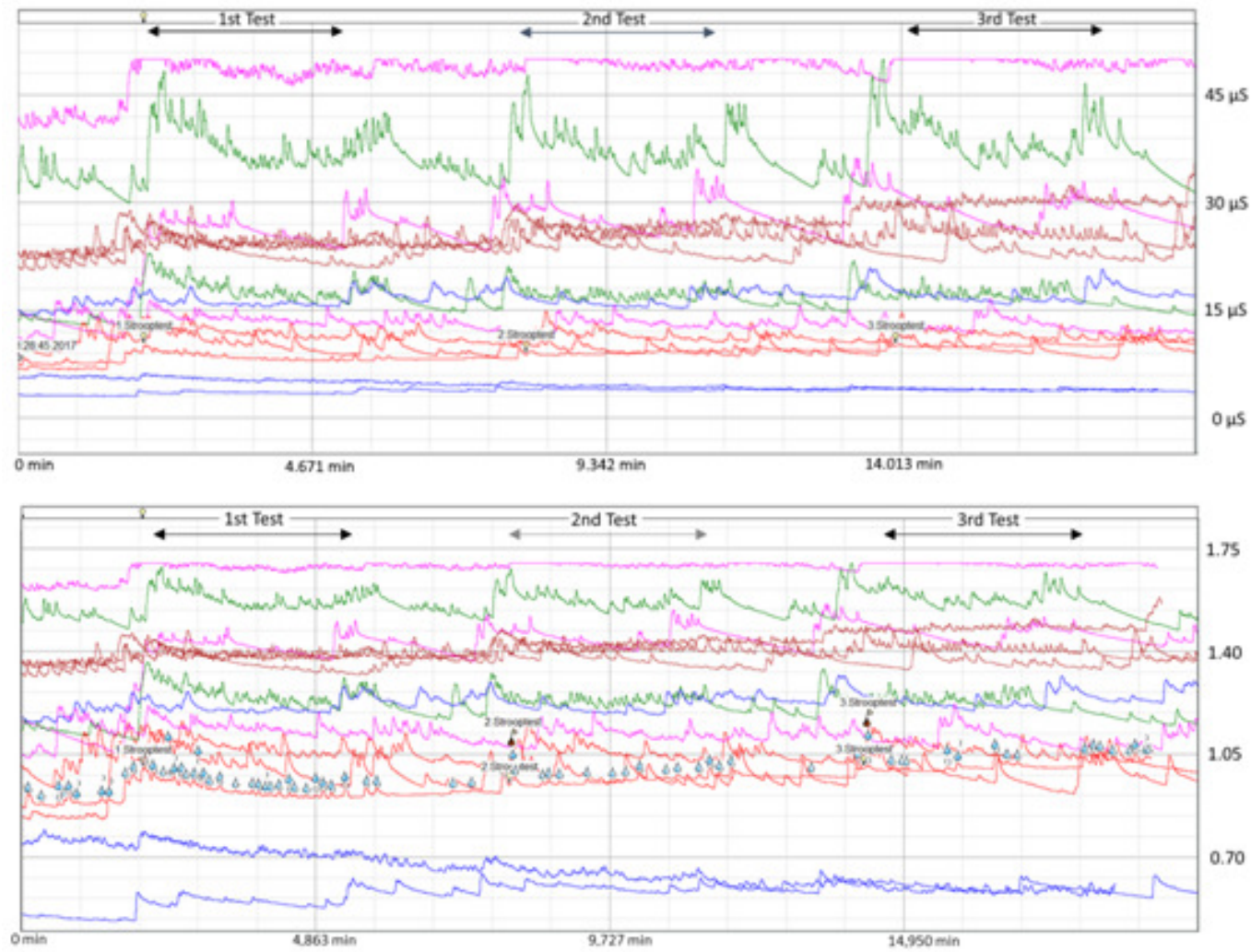

Figure 2. Raw data $(\mu S)$-top- and logarithmized data -bottom- of the repeated Stroop-test over the time (min) of 14 subjects. Between the repetitions two minutes of rest were measured. Starting time is two minutes before the first stress stimuli was set.

Figure 3 shows the first measurement of the Stroop-test after applying the range correction and ztransformation on the SCL data.
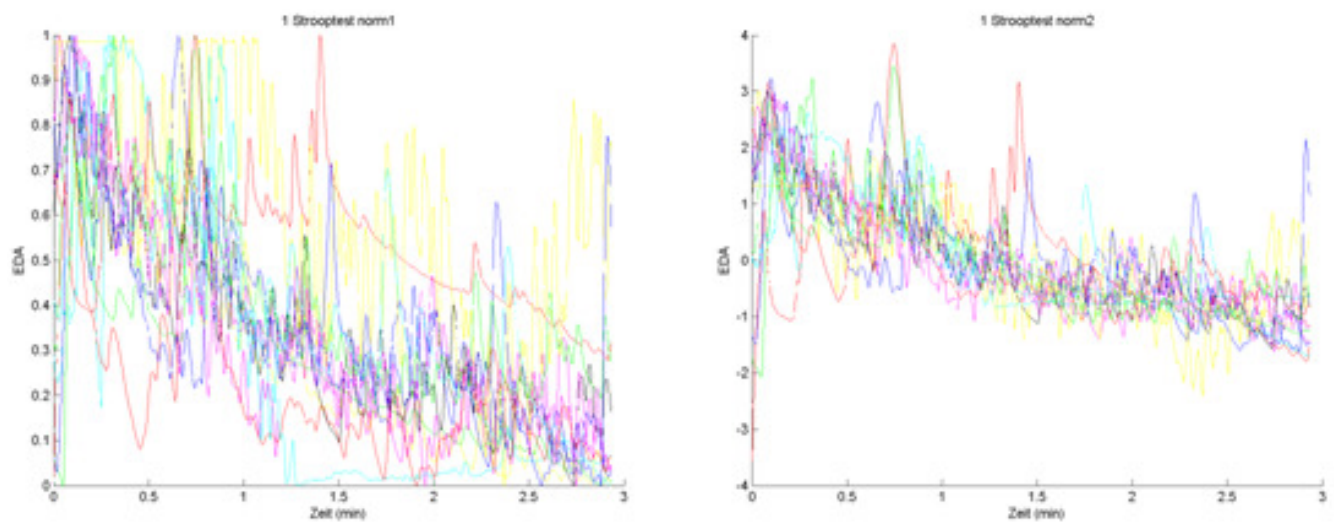

Figure 3. Range correction on the left and z-transformation on the right. The data are from the Stroop-test from 14 subjects. 


\subsection{Intra-individual Comparison}

The logarithmic differentiated SCL data of the baseline and rest measurements before the first stress stimulus differed descriptively in the Stroop-test M_Baseline $_{\text {Stroop }}=.79$,

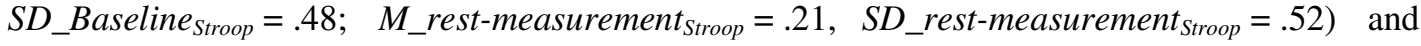
in the cold-pressor-test $\quad\left(M \_\right.$Baseline $_{C P T}=.54, S D \_$Baseline ${ }_{C P T}=.95 ;$ M_restmeasurement $t_{C P T}=.12, S D \_$rest-measurement $\left.{ }_{C P T}=.46\right)$.

A one-way ANOVA with repeated measures shows a significant main effect between all the mean difference values of baseline and rest in both stress tests $(F[3,39]=6.14, p<.05, \varepsilon=.06$, $\eta \mathrm{p}^{2}=.32$ ). The t-test for dependent samples implies a significant difference between the baseline and resting values in the Stroop-test $(t[13]=5.58, p<.01)$, while the difference in the coldpressor test is not significant $(t[13]=1.92, p>.01)$.

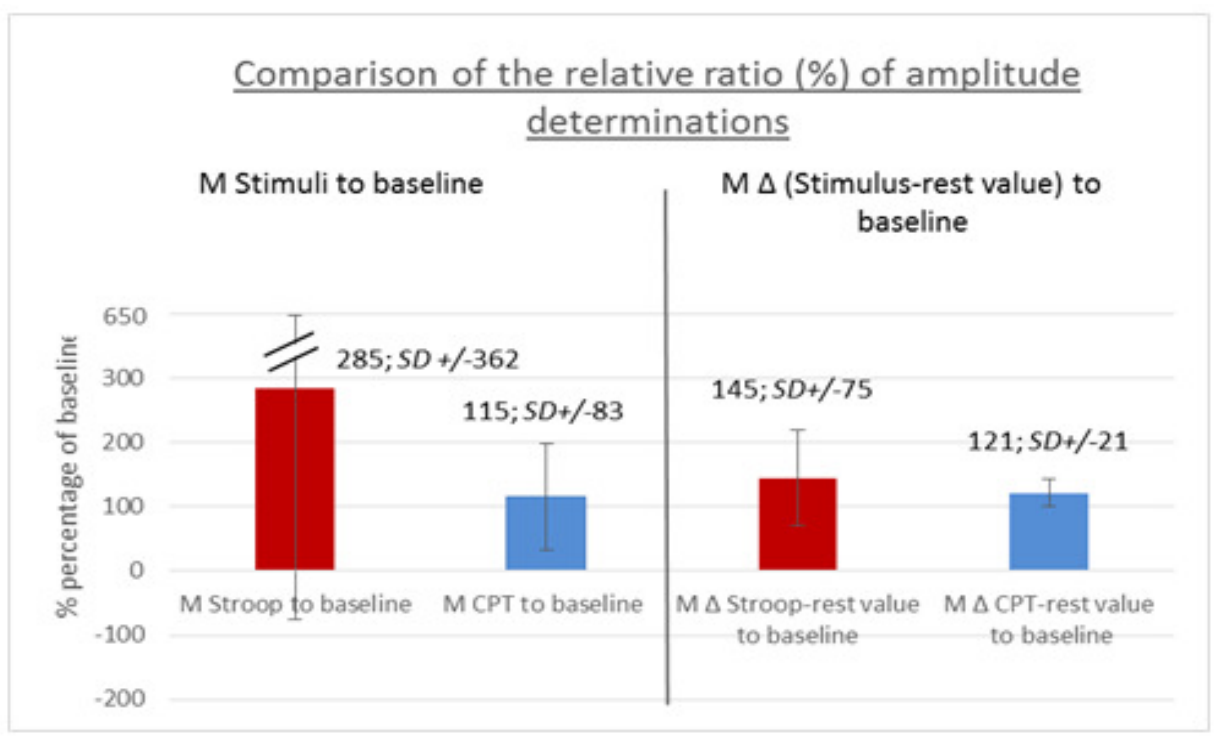

Figure 4. Presentation of the effect of different amplitude determinations. Left: Difference of the mean strain tests to the mean baseline. Right: Correction method by difference of the mean strain tests to the respective rest value ( $M \pm S D, n=3$ repeated measures).

Figure 4 shows two possibilities for the calculation of the effect size of the strain required for the relative ratio calculation to the baseline. Both calculations contain the mean SCL of the three repeated measures in the Stroop- and cold-pressor-tests. On the left, the ratio of the mean strain value to the mean baseline value is shown, while the right side shows the ratio of the mean strain value to the mean baseline value using the correction method. The effect size is corrected by the difference of the immediately previous rest value from the mean strain value. The Stroop-test reveals relative values that are twice as high and five times the standard deviations without using the correction method. The cold-pressor-test displays a reduced variation $17 \%$ instead of a $72 \%$ without the elimination of disturbance factors.

\subsection{Examination of the stress-level, effect size and reliability of the single stress tests}

Descriptively, the three logarithmic repeated measures have a very similar SCL mean value $\left(M \_S C L 1 S t r o o p=1.20, S D \_S C L 1 \_S t r o o p=.32 ; M \_S C L 2 S t r o o p=1.20, S D \_S C L 2 \_S t r o o p=.32\right.$; $M \_S C L 3 S t r o o p=1.20, S D \_S C L 3$ _Stroop $\left.=.34\right)$. The one-way ANOVA shows no significant difference between the repeated measures in the Stroop-test $(F[1.09,14.17]=1.69, p>.05$, 
$\left.\varepsilon=.55, \eta p^{2}=.01\right)$. The Retest-Reliability test indicates a high reliability of the repeated measures by the Pearson correlation coefficient (Table 2).
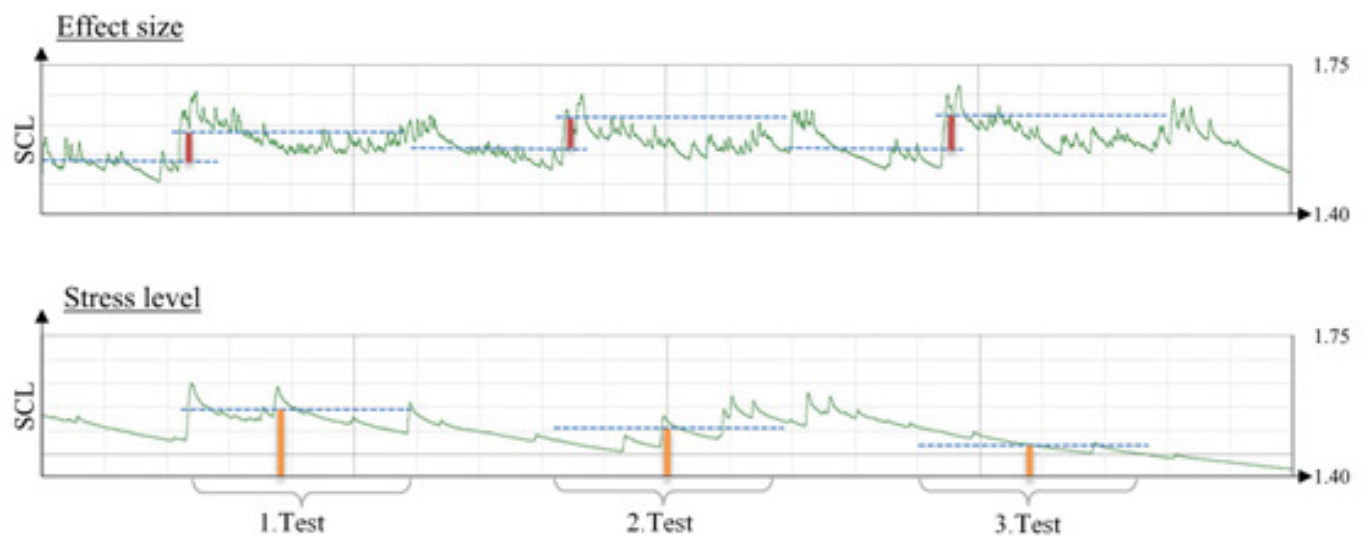

Figure 5. Logarithmic SCL values of a subject in the Stroop-color-word test (top) and cold-pressor-test (bottom). Visual representation of the effect size by determination of the amplitude (difference mean-stress test of mean-rest) (top) and by determination of the absolute mean of the stress test respectively (bottom).

On the contrary, the amplitudes of the SCL have a major effect in the repeated measures $\left(F[2,26]=10.06, p<.05, \varepsilon=.75, \eta \mathrm{p}^{2}=.44\right)$. The $t$-test for differentiated distinction results in a significant effect between the mean values in $M_{-} A m p 1_{\text {Stroop }}$ und $M_{-} \_A m p 2_{\text {Stroop }}(\mathrm{t}[13]=3.11$, $p<.02)$, as well as significance between $M_{-} A m p 1_{\text {Stroop }}$ und $M_{-} \_$Amp $3_{\text {Stroop }}(\mathrm{t}[13]=3.11, p<.02$, but no significant difference between $M_{-} A m p 2_{\text {Stroop }}$ und $M_{-} A m p 3_{\text {Stroop }}(\mathrm{t}[13]=-.19, p>.02)$. The repeated measures of the amplitudes point unreliable based on Pearson's correlation coefficient (Table 2).

The logarithmic absolute SCL mean values of the cold-pressor-test indicate a drop in the repeated measures $\quad\left(M \_S C L 1_{C P T}=1.06, \quad S D \_S C L 1_{C P T}=.35 ; \quad M \_S C L 2_{C P T}=.95, \quad S D \_S C L 2_{C P T}=.41\right.$; $\left.M \_S C L 3_{C P T}=.89, S D \_S C L 3_{C P T}=.43\right)$, which is confirmed by the one-way ANOVA with repeated measures $\left(F[1.07,12.90]=8.70, p<.05, \varepsilon=.54, \eta \mathrm{p}^{2}=.42\right)$.

The examination in the cold-pressor-test with the $t$-test for differentiation results between mean values $M \_S C L 1_{C P T}$ and $M \_S C L 2_{C P T}(t[12]=2.38, p>.02)$ showed no difference, but between $M \_S C L 1_{C P T}$ and $M \_S C L 3_{C P T}(t[12]=3.21, p<.02)$ a significant difference was revealed and as well as between $M \_S C L 2_{C P T}$ and $M \_S C L 3_{C P T}(t[12]=4.55, p<.02)$. Using Pearson's correlation coefficients for reliability established reliability between all three repeated measures. The amplitudes of the SCL show no major difference in mean value $\left(M_{-} A m p I_{C P T}=.12\right.$, $S D \_A m p 1_{C P T}=.47 ; \quad M \_A m p 2_{C P T}=-.12, \quad S D \_A m p 2_{C P T}=.58 ; \quad M \_A m p 3_{C P T}=-.27$, $\left.S D \_A m p 3_{C P T}=.49\right)$. The variance analysis confirms the missing significant difference in mean value $\quad\left(F[12,26]=2.48, \quad p>.05, \quad \varepsilon=.98, \quad \eta p^{2}=.16\right)$. The retest reliability test by means of Pearson's correlation coefficient shows no reliability in the amplitude between all repeated measures (Table 2). 
Table 2. Visualization of the examinations with results from both stress test. Means, standard deviations and correlations shown in the tables are always the logarithmically transformed values. The number in the column "reliability" concerned the repeated measurement.

\begin{tabular}{|c|c|c|c|c|}
\hline & $\begin{array}{l}\text { stress level } \\
\mathrm{M} \pm \mathrm{SD}\end{array}$ & $\begin{array}{l}\text { reliability } \\
\mathrm{r}\end{array}$ & $\begin{array}{l}\text { Effect size } \\
M \pm S D\end{array}$ & $\begin{array}{l}\text { reliability } \\
\mathrm{r}\end{array}$ \\
\hline \multicolumn{5}{|l|}{ Stroop } \\
\hline 1. Test & $1.20 \pm .32$ & between $1 \& 2=.99 * *$ & $.21 \pm .53$ & between $1 \& 2=.50$ \\
\hline 2. $\quad$ Test & $1.20 \pm .32$ & between $2 \& 3=1.00^{* *}$ & $-.21 \pm .42$ & between $2 \& 3=.84^{* *}$ \\
\hline 3. Test & $1.20 \pm .34$ & between $1 \& 3=.99 * *$ & $-.22 \pm .46$ & between $1 \& 3=.50$ \\
\hline \multicolumn{5}{|l|}{ cold-pressor } \\
\hline 1. Test & $1.06 \pm .35$ & between $1 \& 2=.91 * *$ & $.12 \pm .47$ & between $1 \& 2=.32$ \\
\hline 2. $\quad$ Test & $.95 \pm .41$ & between $2 \& 3=1.00^{* *}$ & $-.12 \pm .58$ & between $2 \& 3=.29$ \\
\hline 3. Test & $.89 \pm .43$ & between $1 \& 3=.90^{* *}$ & $-.27 \pm .49$ & between $1 \& 3=-.05$ \\
\hline
\end{tabular}

The correlation tests of the variables identify some few correlations. There is a significant correlation between the SCL rest values and the mean SCL amplitudes in both stress tests (Stroop: $\mathrm{r}=.70 ;$ CPT: $\mathrm{r}=.72$ ) but not between the SCL baseline and the SCL amplitudes. Furthermore, there is a positive significant correlation between the SCL and the number of NS.SCRs in the baseline $(r=.76)$. Neither the amount of the NS.SCRs in the baseline nor the NS.SCRs in the rest measurement had any correlation to the SCL amplitudes in both stress tests. At least the examination of the relative amount of NS.SCRs to the baseline to the relative amplitude value to baseline showed no correlation character.

\section{DISCUSSION}

The aim of the present work was to verify the use of the SCL as an index of sympathetic activation for combined monitoring with blood pressure in hospitals. The emphasis was on the attempt to standardize SCL data to compare the SCL behaviour of different persons and interpret it by comparison with standard values. Additionally, the SCL data in combination with continuous blood pressure values should provide information about a retrospective relationship of the sympathetic function.

The opportunity to use the SCL as an index of sympathetic activation was demonstrated by visible effects of physical as well as psychological stress situations with the SCL. The raw and logarithmic SCL values correspond to the specifications of Venables and Christie [22] for exosomatic measurements with active electrodes. The examinations show a major inter-individual variability, which also exists under predominantly standardized measuring methods and ambient conditions.

The inter-individual comparison showed similar descriptive curves among the subjects, but the standardization methods used could not reduce the variability sufficiently.

As a result of the inter-individual comparison analysis, the use of a correction method was established for the determination of the real strain effect. For this purpose, the difference between the strain value and the immediately preceding rest value is drawn before a relative comparison is made with the baseline. 
International Journal of Biomedical Engineering and Science (IJBES), Vol. 5, No. 2, April 2018

The SCL curve in both stress tests was reliable in the repeated measures, other than the amplitude size, which reflects the effect of the stimulus. This fact leads to the conclusion that although the response pattern among the subjects was similar, the effect of the stimulus was not the same. These results are reflected in the study of Weise and Heinecke [23], which examined the reliability of absolute and relative SCL values only relativized to the rest measurement over a 3month period. The influence of momentary individual states of excitement, such as alertness, attention, the type of instruction, previous habituation, emotional state or motivation, is difficult to determine or control. In other studies, a high variability of the SCL and NS.SCR in the coldpressor-test occurred, but could not be explained so far, even with the help of other parameters $[24,25]$. These facts exclude the use of a stress stimulus as a measure of calibration, because of the strong individual response to excitation processes and the sensitive response of the EDA to any stimulus.

Furthermore, common electrodermal tendencies between tonic parameters (SCL, amplitude, NS.SCRs) were analyzed for the intra-individual examination point. This way, conclusions about the behaviour of tonic parameters among subjects and differences in strain should be made. Only two out of six correlation tests investigated revealed significant correlations for statements on EDA behaviour by tonic parameters. Some other authors confirm the non-normative NS.SCR frequency values $[1,26]$. Accordingly, an intra-individual comparison that refers to the comparison with the baseline value has to be selected for the analysis. For this purpose, the correction method with the amplitude determination should be considered for the immediate rest value. It offers the advantage of determining the real effects of sympathetic activation. Important to note, is that a classification of the individual SCL range is only possible via experience with implication of maximum and minimum SCL values, since a calibration measure is difficult to implement by habituation effects.

Nevertheless, a possible limitation of the present test series is the absence of standardization of the environmental conditions. The questionnaire revealed some disruptive factors for example direct acoustic factors, such as traffic noise as well as indirect disturbance factors, which were due to the presence of the principal investigator. Another limitation is the sample size of 15 subjects that were young and had a non-homogeneous gender distribution. With this amount the generalizability of the results is reduced for the total population.

The reliability measurement was limited to a triple measurement repetition at one day. Because the EDA measurement is depending on the psychological state of a person, several repeated measurements over a longer period would be more significant for an intra-individual comparison. It is to consider, that the longer the time period of retesting, the lower the stability of the physiological measure. In addition to the EDA, further vegetative parameters should be used for a meaningful analysis of blood pressure changes. The very fact that in hypotonic patients the dependence of the EDA on the blood pressure is inconsistent [27, 28], the exclusive use of EDA is irresponsible. Additional vegetative parameters would help to make the evaluation of the individual EDA data more precise to offer a coherent overall picture of the sympathetic activity in addition to the EDA measurement.

\section{CONCLUSION}

The attempt to standardize SCL measurements for comparison with standard values was not successful. Although SCL works as an indicator for physical and psychological stress situations the standardization methods applied did not reduce the variability in a sufficient way. Furthermore, calibration measurements are difficult to establish in the intended setting. Different momentary states of excitement, such as alertness, attention, or emotional state influence the measurements and are difficult to determine. Any attempt for generalization needs a bigger 
International Journal of Biomedical Engineering and Science (IJBES), Vol. 5, No. 2, April 2018

sample and longer measurements. EDA as an exclusive tool is not recommended and should always be combined with other parameters.

\section{REFERENCES}

[1] Gesundheitsberichterstattung des Bundes, Sterbefälle (absolut, Sterbeziffer, Ränge, Anteile) für die 10/20/50/100 häufigsten Todesursachen (ab 1998). Gliederungsmerkmale: Jahre, Region, Alter, Geschlecht, ICD-10.

[2] K. H. (. Wesseling, "Finapres - Kontinuierliche, nichtinvasive arterielle Blutdruckmessung am Finger nach der Methode von Peñáz," in Meyer-Sabellek, Gotzen (ed)- Indirekte 24-Stunden Blutdruckmessung, pp. 45-59.

[3] W. Boucsein, (2012) Electrodermal Activity. Boston: Springer.

[4] W. Boucsein, (1988) Elektrodermale Aktivität: Grundlagen, Methoden und Anwendungen. Berlin, Heidelberg: Springer.

[5] R. Edelberg, (1967) "Electrical properties of the skin", Methods of psychophysiology, C. C. Brown, Ed., Baltimore: Williams and Wilkens, pp. 1-53.

[6] P. H. Venables and D. A. Mitchell, (1996) "The effects of age, sex and time of testing on skin conductance activity”, (eng), Biological psychology, Vol. 43, No. 2, pp. 87-101.

[7] M. Barontini, J. O. Lazzari, G. Levin, I. Armando, and S. J. Basso, (1997) "Age-related changes in sympathetic activity: biochemical measurements and target organ responses", (eng), Archives of gerontology and geriatrics, Vol. 25, No. 2, pp. 175-186.

[8] M. E. Dawson, A. M. Schell, D. L. Filion, and G. G. Berntson, (2007) "The Electrodermal System”, Handbook of psychophysiology, J. T. Cacioppo, L. G. Tassinary, and G. G. Berntson, Eds., 3rd ed., Cambridge [England], New York: Cambridge University Press, pp. 157-181.

[9] K. S. McNeal, J. M. Spry, R. Mitra, and J. L. Tipton, (2014) "Measuring Student Engagement, Knowledge, and Perceptions of Climate Change in an Introductory Environmental Geology Course", Journal of Geoscience Education, Vol. 62, No. 4, pp. 655-667.

[10] A. M. Schell, M. E. Dawson, K. H. Nuechterlein, K. L. Subotnik, and J. Ventura, (2002) "The temporal stability of electrodermal variables over a one-year period in patients with recent-onset schizophrenia and in normal subjects", Psychophysiology, Vol. 39, No. 2, pp. 124-132.

[11] M. El-Sheikh, (2007) "Children's skin conductance level and reactivity: Are these measures stable over time and across tasks?", (eng), Developmental psychobiology, Vol. 49, No. 2, pp. 180-186.

[12] M. Bullinger et al., (1984) "Endocrine effects of the cold pressor test: relationships to subjective pain appraisal and coping”, (eng), Psychiatry Research, Vol. 12, No. 3, pp. 227-233.

[13] M. Pascualy et al., (2000) "Hypothalamic pituitary adrenocortical and sympathetic nervous system responses to the cold pressor test in Alzheimer's disease", (eng), Biological psychiatry, Vol. 48, No. 3, pp. 247-254.

[14] J. R. Stroop, (1933) "Studies of Intereference in Serial Verbal Reactions", Journal of Experimental Psychology, No. 18, pp. 643-662.

[15] A. Lenhard, (2010) "Stroop-Effekt". 
International Journal of Biomedical Engineering and Science (IJBES), Vol. 5, No. 2, April 2018

[16] J. J. Braithwaite, D. G. Watson, J. Roberts, and R. Mickey, "A Guide for Analysing Electrodermal Activity (EDA) \& Skin Conductance Responses (SCRs) for Psychological Experiments: Technical Report. Selective Attention \&Awareness Laboratory (SAAL), Behavioural Brain Sciences Centre, School of Psychology.

[17] D. T. Lykken and P. H. Venables, (1971) "Direct measurement of skin conductance: A proposal for standardization," Psychophysiology, Vol. 8, No. 5.

[18] D. T. Lykken, (1972) "Range Correction Applied to Heart Rate and to GSR Data", Psychophysiology, Vol. 9, No. 3, pp. 373-379.

[19] D. T. Lykken, R. Rose, B. Luther, and M. Maley, (1966) "Correcting psychophysiological measures for individual differences in range”, Psychological bulletin, No. 66, pp. 481-484.

[20] G. Ben-Shakhar, (1985) "Standardization within individuals: A simple method to neutralize individual differences in skin conductance", Psychophysiology, Vol.22, No. 3, pp. 292-299.

[21] J. Schwarz and H. Bruderer Enzler, Methodenberatung in der Statistik. Accessed on: May 042017.

[22] P. H. Venables and M. J. Christie, (1980) "Elektrodermal activity", Techniques in psychophysiology, I. Martin and P. H. Venables, Eds., Chichester: Willey, pp. 3-67.

[23] C. Weise, K. Heinecke, and W. Rief, (2008) "Stability of physiological variables in chronic tinnitus sufferers", (eng), Applied psychophysiology and biofeedback, Vol. 33, No. 3, pp. 149-159.

[24] H. F. Posada-Quintero et al., (2016) "Power Spectral Density Analysis of Electrodermal Activity for Sympathetic Function Assessment”, Annals of Biomedical Engineering, No. April, pp. 1-12.

[25] G. H. Prystav, (1976) "Electrodermal, cardiac, and respiratory activity to repeated cold pressor stimulation in drug addicts," (eng), The Journal of general psychology, Vol. 94, No. 2d Half, pp. 259270.

[26] J. Fahrenberg, (1979) „Psychophysiologische Aktivierungsforschung: Ein Beitrag zu den Grundlagen der multivarianten Emotions- und Stress-Theorie.“, München: Minerva.

[27] E. Kronholm, M. T. Hyyppä, A. Jula, and T. Toikka, (1996) "Electrodermal lability and hypertension”, International Journal of Psychophysiology, Vol. 23, No. 1-2, pp. 129-136.

[28] R. M. Kelsey, (1991) "Electrodermal lability and myocardial reactivity to stress", (eng), Psychophysiology, Vol. 28, No. 6, pp. 619-631. 